\title{
The first K2 roAp star: HD 24355 pulsating in a distorted quadruploe mode
}

\author{
Daniel L. Holdsworth ${ }^{1, \star}$, Donald W. Kurtz ${ }^{1}$, Barry Smalley ${ }^{2,}$, Hideyuki Saio ${ }^{3}$, Gerald Handler $^{4}$, Simon J. Murphy ${ }^{5}$, \\ and Holger Lehmann 6 , \\ ${ }^{1}$ Jeremiah Horrocks Institute, University of Central Lancashire, Preston, PR1 2HE, UK \\ ${ }^{2}$ Astrophysics Department, Keele University, Staffordshire, ST5 5BG, UK \\ ${ }^{3}$ Astronomical Institute, School of Science, Tohoku University, Sendai 980-8578, Japan \\ ${ }^{4}$ Copernicus Astronomical Center, Bartycka 18, PL-00-716 Warsaw, Poland \\ ${ }^{5}$ Sydney Institute for Astronomy (SIfA), School of Physics, University of Sydney, NSW 2006, Australia \\ ${ }^{6}$ Thüringer Landessternwarte Tautenburg (TLS), Sternwarte 5, D-07778 Tautenburg, Germany
}

\begin{abstract}
We present an analysis of the first K2 observations of a rapidly oscillating Ap (roAp) star, HD 24355. The star was discovered to be a roAp star by [1], with a frequency of $224.31 \mathrm{~d}^{-1}(2596.18 \mu \mathrm{Hz} ; \mathrm{P}=$ $6.4 \mathrm{~min}$ ) and an amplitude of $1.51 \mathrm{mmag}$ in SuperWASP broadband photometry. Spectroscopic analysis of low-resolution spectra show the star to be an A5 Vp SrEu star. The high precision K2 data allow us to identify 13 rotationally split sidelobes to the main pulsation frequency. This is the largest number of sidelobes seen in a roAp star to date. We also see an unusual pulsational phase variation as the star rotates, showing this star to be the most distorted quadrupole roAp pulsator yet observed. Modelling of this star confirms its quadrupole nature, and allows us to constrain the magnetic field strength, angle of inclination and the angle of obliquity.
\end{abstract}

\section{Introduction}

The rapidly oscillating Ap (roAp) stars are a rare subclass of the chemically peculiar, magnetic, Ap stars. They show pulsations in the range of $6-23$ min with amplitudes up to $18 \mathrm{mmag}$ in Johnson $B$ [2], and are found at the base of the classical instability strip on the Hertzsprung-Russell (HR) diagram, from the zero-age main-sequence to the terminal-age main-sequence in luminosity. Since their discovery by [3], only 61 of these objects have been identified (see [4] for a catalogue). The pulsations are high-overtone pressure modes (p modes) thought to be driven by the $\kappa$ mechanism acting in the $\mathrm{H}_{\mathrm{I}}$ ionisation zone [5]. However, [6] have shown that turbulent pressure in the convective zone may excite some of the modes seen in a selection of roAp stars.

The pulsation axis of these stars is inclined to the rotation axis, and closely aligned with the magnetic one, leading to the oblique pulsator model [e.g. 3, 7-11]. Oblique pulsation allows the pulsation modes to be viewed from varying aspects over the rotation cycle of the star, giving constraints on the pulsation geometry that are not available for any other type of pulsating star (other than the Sun, which is uniquely resolved).

The mean magnetic field modulus in Ap stars are strong, of the order of a few $\mathrm{kG}$ to $34 \mathrm{kG}$ [12]. A strong magnetic field suppresses convection and provides stability to allow radiative levitation of some elements producing a stratified atmosphere with surface inhomogeneities.

\footnotetext{
^e-mail: dlholdsworth@uclan.ac.uk
}

These inhomogeneities, or spots, are long lasting (decades in many known cases) on the surface of Ap stars, thus allowing for an accurate determination of the rotation period of the star. In the spots rare earth elements such as $\mathrm{La}, \mathrm{Ce}$, Pr, Nd, Sm, Eu, Gd, Tb, Dy and Ho, may be overabundant by up to a million times the solar value, leading to spectral line strength variations over the rotation period [e.g. 13]. Because of the complex atmospheres of the Ap stars, the roAp stars provide the best laboratory, beyond the Sun, to study the interactions between pulsations, rotation, and chemical abundances in the presence of magnetic fields.

Early photometric campaigns targeted known Ap stars in the search for oscillations [e.g. 14, 15], with later studies using high-resolution spectroscopy to detect line profile variations in Ap stars caused by pulsational velocity shifts [e.g. 16-21]. Most recently, the use of the SuperWASP [Wide Angle Search for Planets; 22] ground-based photometric survey led to the identification of 11 roAp stars $[1,2]$.

With the launch of the Kepler space telescope, the ability to probe to $\mu \mathrm{mag}$ precision has enabled the detection of four roAp stars with amplitudes below the ground-based detection limit [4, 23-25], and the analysis of a further known roAp star [26].

The re-purposing of the Kepler spacecraft after the failure of the second reaction wheel has opened up a host of new possibilities to observe variable stars at $\mu$ mag precision. Here we present the first observations, in Short Cadence, of a classical, high-amplitude, roAp star. 
HD 24355 is a bright $(V=9.65)$ rapidly oscillating Ap star, discovered by [1]. Their data show a pulsation at $224.31 \mathrm{~d}^{-1}(2596.18 \mu \mathrm{Hz} ; P=6.4 \mathrm{~min})$ with an amplitude of $1.51 \mathrm{mmag}$ in the WASP broadband filter. We present here spectral classification followed by a discussion of the SuperWASP discovery data, alongside further ground-based observations. We then present an analysis of the K2 Campaign 4 data.

\section{Spectral Classification}

We obtained two classification resolution spectra of HD 24355 (Fig. 1). The first with the Intermediate dispersion Spectrograph and Imaging System (ISIS) mounted on the 4.2-m William Herschel Telescope (WHT), at a resolution of $R \sim 2000$. The second spectrum was obtained using the Wide-Field Spectrograph (WiFeS) mounted on the 2.3-m Australian National Observatory (ANU) telescope at a resolution of $R \sim 3000$.

In addition, HD 24355 has been observed as part of the LAMOST campaign $[27,28]$ and was made public as part of Data Release 1 [DR1; 29]. The LAMOST spectrum was obtained at a resolution of $R \sim 1800$, and is shown in Fig. 1 alongside the other spectra. Furthermore, in addition to classification spectra, we have obtained high-resolution, $R \sim 58000$, spectra using the coudé échelle spectrograph mounted on the 2.0-m telescope at the Thüringer Landessternwarte (TLS) Tautenburg, Germany.

Following the classification process described by [30], we see that the Balmer line types are close matches to the A5 and A7 MK standard stars ${ }^{1}$ (HD 23194 and HD 23156, respectively) with many of the metal lines being well matched by the standard A5 star. There are, however, peculiarities which do not match the standard stars. All spectra show strong enhancements of Sr II and Eu II. While the Eu II line at $4130 \AA$ can be blended with a Si II doublet at 4128 and $4131 \AA$, we see no sign of Si II at 3856 and $3862 \AA$. Lines of $\mathrm{Cr}$ II are not enhanced when compared to the MK standard stars, ruling out $\mathrm{Cr}$ peculiarities. With the obvious presence of Sr II and Eu II in the spectra, we conclude that HD 24355 is an A5Vp SrEu star.

We derive an effective temperature for HD 24355 by fitting the Balmer lines of our spectra. Given their low resolution, we are unable to determine a $\log g$ of the star, so fix this value at 4.0 which is a reasonable approximation for this exercise. From the WiFeS spectrum we derive $T_{\text {eff }}=8200 \pm 250 \mathrm{~K}$ and from the ISIS data we find $T_{\text {eff }}=8200 \pm 200 \mathrm{~K}$.

The LAMOST spectra are automatically analysed by instrument specific software to determine fundamental parameters. For HD 24355 the pipeline suggests $T_{\text {eff }}=$ $8638 \pm 100 \mathrm{~K}, \log g=4.15 \pm 0.41(\mathrm{cgs})$ and $[\mathrm{Fe} / \mathrm{H}]=$ $0.7 \pm 0.22$. However, when trying to fit these parameters to the spectrum, we find a large disparity. Given the resolution of the spectrum, we are unable to determine a revised $\log g$ (so we set this to 4.0 as above). We find

\footnotetext{
${ }^{1}$ Spectra of the standard stars were obtained from R.O. Gray's website: http://stellar.phys.appstate.edu/Standards/std1_8.html
}

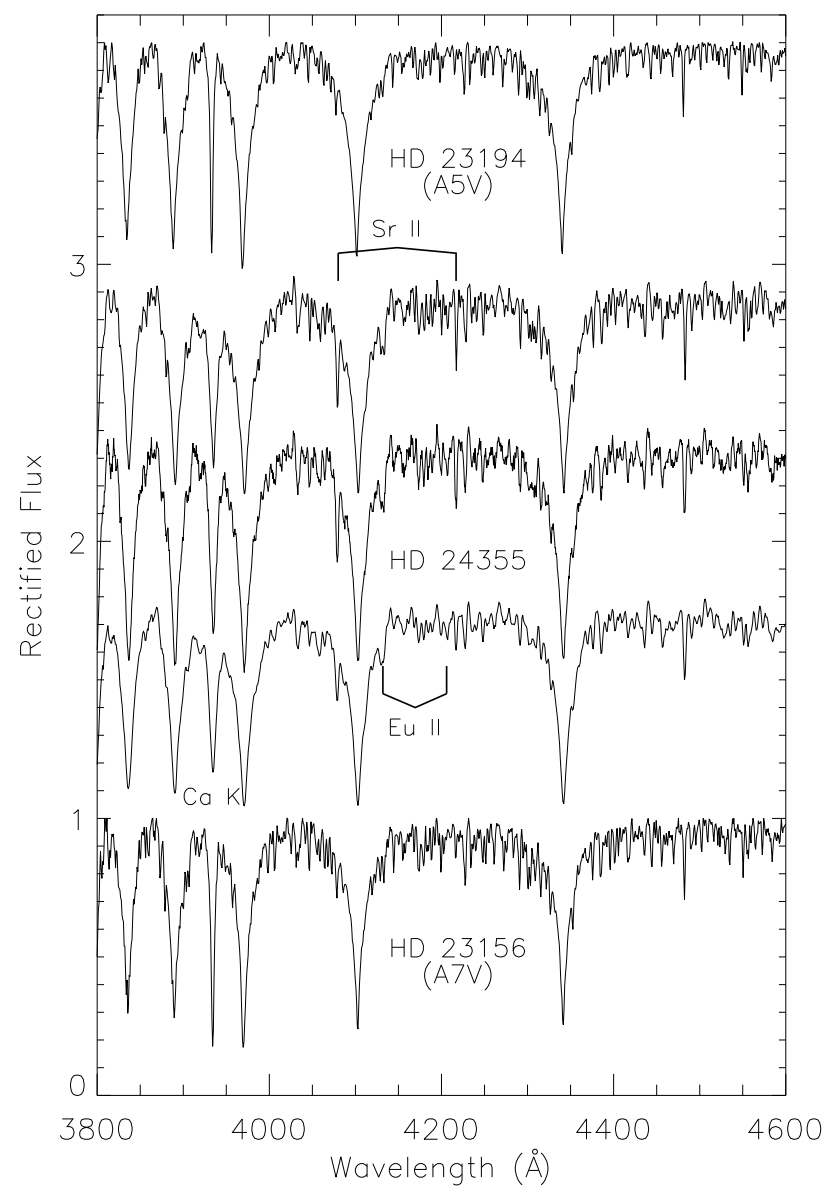

Figure 1: Spectra of HD 24355 (the three central spectra) compared with two MK standard stars of A5 and A7 type. Note the strong lines of $\mathrm{Sr}$ II at 4077 and $4216 \AA$ and the enhanced lines of Eu II at 4130 and $4205 \AA$ in the target star. The upper spectrum for HD 24355 was obtained with the ANU/WiFes instrument, the second with the WHT/ISIS instrument, with the lower obtained with the LAMOST instrument. The spectra have been ordered in rotation phase and offset for clarity.

that $T_{\text {eff }}=8150 \pm 250 \mathrm{~K}$ fits the Balmer lines much better than the higher value of the LAMOST estimate, and $8150 \pm 250 \mathrm{~K}$ is in agreement with the other two spectra.

For further details on the spectral analysis of HD 24355, we refer the reader to [31].

\section{Photometry}

The pulsation in HD 24355 was discovered by [1]. Here we provide an overview of the discovery data, with the addition of further ground-based data obtained with the 0.75-m Automatic Photoelectric Telescope (APT) at Fairborn Observatory in Arizona, USA. Our main analysis is of the first Short Cadence (SC) data of a roAp star from the re-purposed Kepler space telescope, K2. 


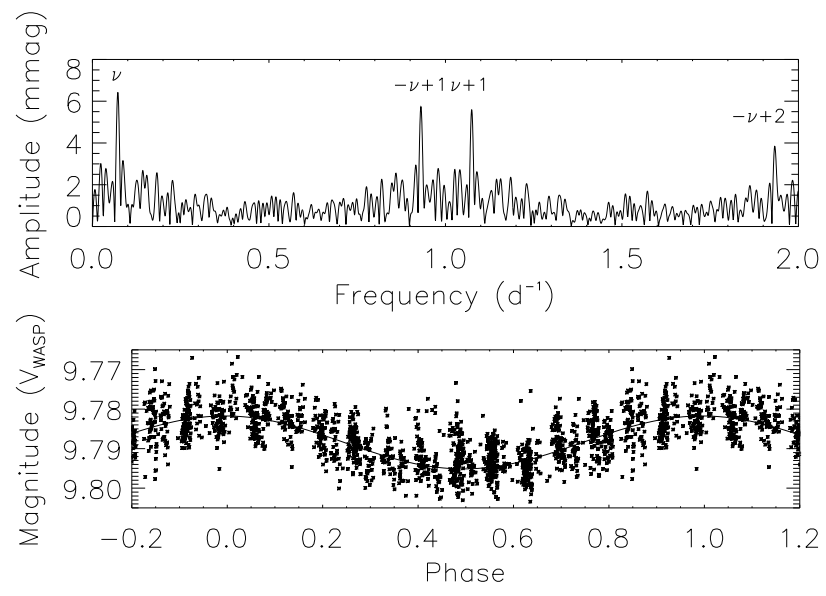

Figure 2: Top: low-frequency periodogram of the SuperWASP light curve from 2010 showing what was thought to be the rotation frequency of the star, as well as its positive and negative aliases. Bottom: SuperWASP phase folded light curve on the frequency determined in the upper panel, i.e. $v 0.07165 \mathrm{~d}^{-1}$. The data have been binned to $5: 1$.

\subsection{Ground-based observations}

\subsubsection{SuperWASP}

SuperWASP observed HD 24355 for three seasons, 2006, 2009 and 2010. The data were following the procedure described by [1].

The light curve shows a clear signature at low frequency that is indicative of rotation (Fig. 2). When all seasons of WASP data are combined to calculate the rotation period, there is evidence of a sub-harmonic to that frequency. We therefore simultaneously calculate the peak at $0.0716 \mathrm{~d}^{-1}$ and a frequency at half that value, namely $0.0358219 \mathrm{~d}^{-1}$. In doing so, we are able to find a more precise measure of the rotation frequency and its harmonic. We find, $v=0.0716439 \pm 0.0000063 \mathrm{~d}^{-1}$ and $v / 2=0.0358220 \pm 0.0000032$, corresponding to periods of $13.9579 \pm 0.0011 \mathrm{~d}$ and $27.9158 \pm 0.0025 \mathrm{~d}$, respectively. In this ground-based survey data, differentiating between the rotation frequency and any harmonics can be problematic. We therefore confirm the true rotation frequency with the $\mathrm{K} 2$ data, in section 3.2.

The pulsation signature of HD 24355 is apparent in all seasons of data, and is shown in Fig. 3. The pulsation is clearly seen at a frequency of $224.3071 \mathrm{~d}^{-1}$ with an amplitude of $1.51 \mathrm{mmag}$ in the WASP passband.

Due to the survey nature of this ground-based data set, not much information can be extracted: the noise level in the high-frequency range is too great to discern, with confidence, sidelobes of the pulsation split by the rotation frequency. Analysis of the $\mathrm{K} 2$ data will provide more precise results on the pulsation and rotation frequencies.

\subsection{2 $0.75-m$ APT}

We observed HD 24355 for three hours on each of three consecutive nights, 2013 November 6, 7, 8, with the 0.75-

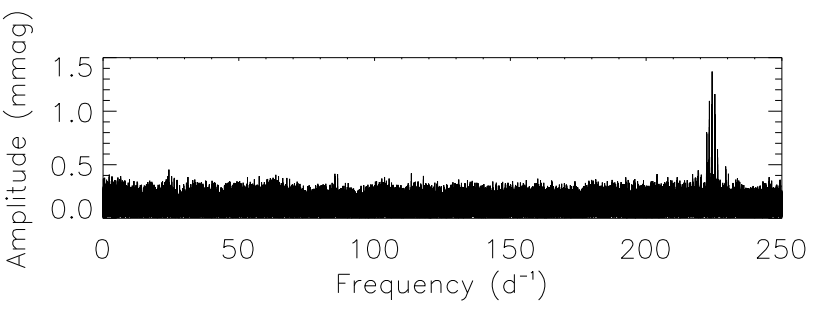

Figure 3: Periodogram of all seasons of WASP data. The data have been pre-whitened to $10 \mathrm{~d}^{-1}$ to the approximate noise level of the high-frequency range to remove the rotation signature and low-frequency noise. Note that the structure surrounding the pulsation signature is a result of daily aliases.

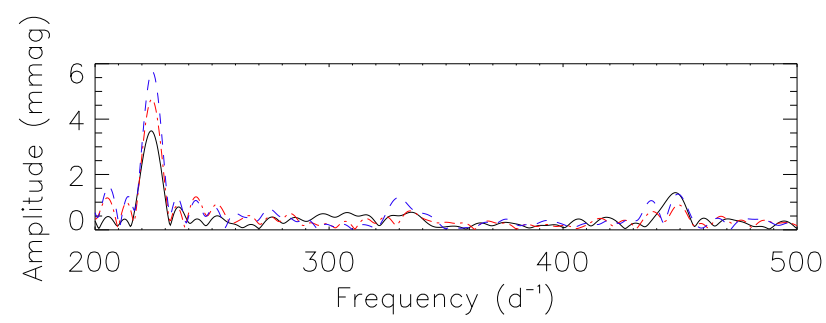

Figure 4: Periodograms of the three nights of APT data. There is a clear increase in the pulsation amplitude as the nights progress, from the solid black line, to the dot-dashed red line, to the dashed blue line. Also evident is the harmonic at twice the pulsation frequency.

m APT installed at Fairborn Observatory, with integrations times of 20-s through a Johnson $B$ filter.

The periodograms for each night of data presented in Fig. 4 show a clear increase in pulsation amplitude over the three nights that the target was observed. This is expected from the oblique pulsator model [3, 11], and is also observed in the $\mathrm{K} 2$ observations (see section 3.2). As the pulsation axis is often mis-aligned with the rotation axis in the roAp stars, the pulsation is viewed from varying aspects over the rotation period, giving rise to the amplitude variations we see in HD 24355.

\subsection{K2 observations}

HD 24355 was observed as part of Campaign 4 (proposal number GO4014) of the K2 mission. The observations covered a period of $70.90 \mathrm{~d}$, and were reduced manually using the PyKE tools provided by the K2 Guest Observer office [32]. The final light curve is shown in the top panel of Fig. 5.

\subsubsection{Rotation signature}

Despite the thruster firings still being present in the data, the rotation signature is clearly seen in Fig. 5. The doublewave nature of the light curve suggests we see both magnetic poles of the star, where the chemically peculiar spots form. The K2 data allow us to unambiguously determine the rotation period of the star. 


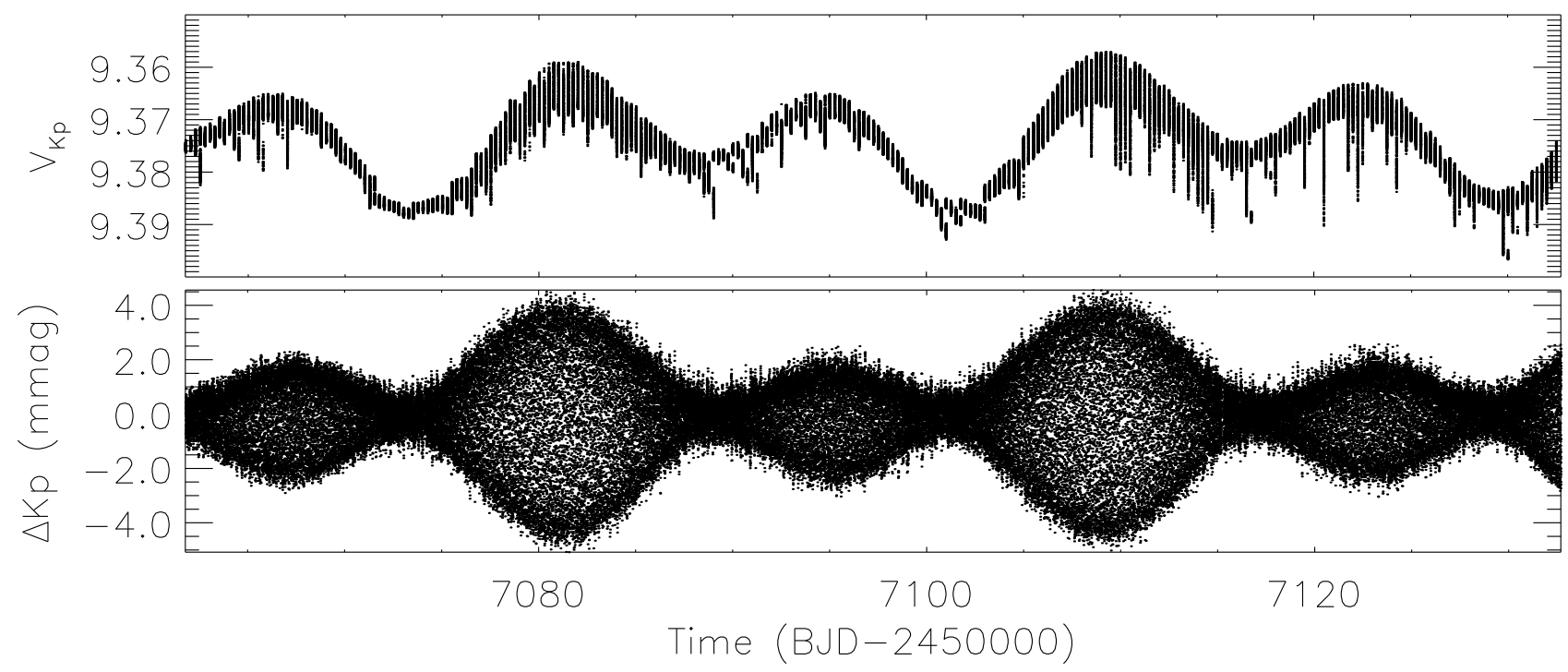

Figure 5: Top: full K2 light curve after data reduction and cleaning has been performed. Clearly evident is the rotational signal, as well as the spacecraft motions. Bottom: light curve of HD 24355 after removal of the rotation signature, and cleaning of low-frequency peaks from the periodogram; these include the harmonics of the thruster frequency. The pulsation envelope is evident, with pulsational maximum occurring at the same time as rotation light maximum within the errors.

Using PerIOD04 we calculate an amplitude spectrum to $0.5 \mathrm{~d}^{-1}$. The result shows two peaks, corresponding to the two consecutive dimmings in the light curve. The principal peak is found at a frequency of $0.071642 \pm 0.000011 \mathrm{~d}^{-1}$, with the secondary at $0.036121 \pm$ $0.000019 \mathrm{~d}^{-1}$, as seen in the WASP data. Fig. 6 shows the light curve phased on the lower frequency. This is clearly the correct rotation frequency. Due to the relatively short length of the $\mathrm{K} 2$ data (covering $\sim 2.5$ rotation periods), we take the period derived from the WASP data (which covers $\sim 57.3$ rotation periods) as the rotation period for HD 24355, namely $P=27.9158 \pm 0.0025 \mathrm{~d}$.

The rotation phases are calculated according to:

$$
\phi(E)=2457108.8867+27.9158 \times E,
$$

where $E$ is the number of rotation cycles elapsed since the reference time, and the reference time is the second pulsational maximum in the $\mathrm{K} 2$ data set.

\subsubsection{Pulsation signature}

Clearly evident in the K2 periodogram of HD 24355 is the pulsation detected by both the WASP and APT observations. To study the pulsation in detail, we remove the rotation signature from the light curve, and significant peaks in the low-frequency domain. This process results in the light curve shown in the bottom panel of Fig. 5.

Fig. 7 shows the pulsation at a frequency of $224.3043 \mathrm{~d}^{-1}$ at an amplitude of $1.74 \mathrm{mmag}$, and four of the sidelobes of the principal peak after it has been removed. Removing the four peaks shown in Fig. 7 reveals further power at frequencies split by ever increasing multiples of the rotation frequency. In total, we identify 13 rotational sidelobes $\left(v+7 v_{\text {rot }}\right.$ is below the noise level), the

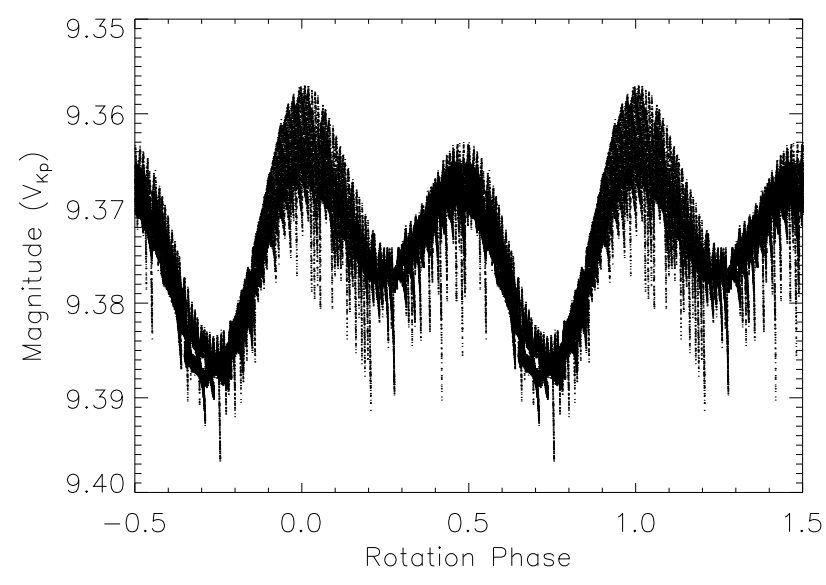

Figure 6: K2 phased light curve, phased on a period of $27.9166 \mathrm{~d}$. The phases have been calculated according to equation (1).

most ever seen in a roAp star. See [31] for a graphical representation of the pre-whitening of the sidelobes.

The oblique pulsation model expects that the frequencies of the sidelobes are exactly split by the rotation frequency. Therefore, using the rotation period derived from the spot variations, we force the frequencies to be equally split, and perform a linear least-squares fit to the data. When performing this test, we set the zero-point in time such that the phases of the first sidelobes are equal. When the exact splitting of the sidelobes is set, we see that the phases of the central five peaks are similar. For a pure quadrupole mode, we expect a quintuplet of frequencies with all phases equal at the time of pulsation maximum. Since this is not the case here for the phases of the quintuplet frequencies, and because there are further rotational sidelobes, we conclude that the mode is distorted. 


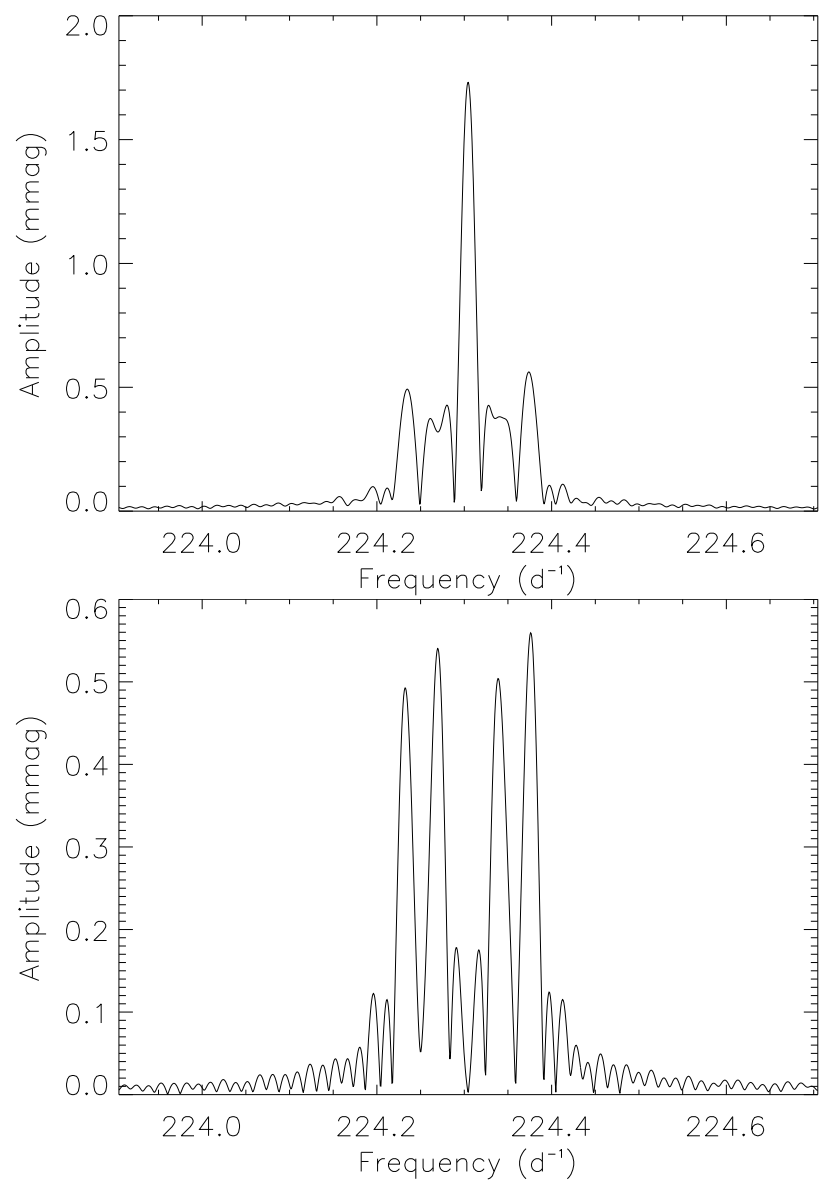

Figure 7: Top: the pulsation signature in the K2 SC data which shows a quintuplet separated by the rotation frequency. Bottom: the rotational sidelobes after the central peak, which is the actual pulsation frequency, has been removed. Analysis of the sidelobe amplitudes informs us on the geometry of the star (see text). Note the amplitude change between the two panels.

\subsection{Frequency variability and a rotational view of the pulsation geometry}

Many of the roAp stars that have been observed at such a high photometric precision as that afforded by the Kepler spacecraft have shown significant variations in their pulsation frequencies [e.g. 4, 26]. To this end, we investigate this phenomenon in HD 24355 by splitting the data into sections of 100 pulsation cycles, or about $0.45 \mathrm{~d}$.

By calculating the frequencies, amplitudes and phases of the pulsation in these sections of data, we are able to monitor the variations of the pulsation over the rotation period of the star. The top-left panel in Fig. 8 shows the variation of the pulsation amplitude over the rotation period of the star. As is expected from the oblique pulsator model, the amplitude varies over the rotation cycle, as the pulsation mode is seen from varying aspects. The amplitude does not reach zero. If the mode were a pure mode, the amplitude would go to zero whenever the line-of-sight passes over a pulsation node; as this is not the case here, we can see that the mode is distorted.

The phase variations seen in the top-right panel of Fig. 8 are very different to those seen in other roAp stars (lower panels of Fig. 8). When the line-of-sight passes over a node, the oblique pulsator model predicts a $\pi$-rad phase shift for a pure dipole or quadrupole mode (as is seen in the other stars). This is not the case for HD 24355, supporting the conclusion that it is a distorted pulsator.

\subsubsection{Mode geometry constraints}

As the $\mathrm{K} 2$ data have a much higher precision than groundbased data, we can derive parameters of the geometry of the pulsation mode using the ratios of the sidelobes following the methodology of [33]. Initially, we test the phase relations between the pulsation mode and the first rotational sidelobes by forcing the sidelobes to have the same phase. We find that $\phi_{-1}=\phi_{+1} \neq \phi_{0}$, suggesting that the mode is distorted.

The clear frequency quintuplet strongly suggests that the pulsation is quadrupolar. We therefore examine the axisymmetric quadrupole case, where $l=2$ and $m=0$, applying a relationship from [33] for a non-distorted oblique quadrupole pulsator in the absence of limb-darkening and spots, i.e.

$$
\tan i \tan \beta=4 \frac{A_{+2}^{(2)}+A_{-2}^{(2)}}{A_{+1}^{(2)}+A_{-1}^{(2)}},
$$

where $i$ is the rotational inclination angle, $\beta$ is the angle of obliquity between the rotation and magnetic axes, and $A_{ \pm 1}^{(2)}$ and $A_{ \pm 2}^{(2)}$ are the amplitudes of the first and second sidelobes of the quadrupole pulsation, respectively. Using the amplitudes from the periodogram, and substituting them into equation (2), we derive $\tan i \tan \beta=3.950 \pm 0.012$ for this simplified case.

We investigate the values of $i$ and $\beta$ that satisfy the constraint of equation (2). All possible values sum to greater than $90^{\circ}$, which is consistent with the rotational light modulation under the assumption that there are spots at both magnetic poles. From our spectra we are able to place an upper limit of $v \sin i \leq 3.5 \mathrm{~km} \mathrm{~s}^{-1}$. Using this value, the rotation period of $27.9158 \mathrm{~d}$ and the derived radius of $R=2.53 \mathrm{R}_{\odot}$, we can estimate an upper limit of $i \leq 50^{\circ}$.

\section{Modelling the amplitude and phase modulation}

Spectroscopic parameters of HD 24355 are $\left(T_{\text {eff }}, \log g\right)=$ $(8200 \pm 200 \mathrm{~K}, 4.0 \pm 0.2)$. Within the error-box we searched for models that have a frequency similar to $224.30 \mathrm{~d}^{-1}$ and reproduce the amplitude and phase modulation.

For each model we picked a non-magnetic quadrupole mode having a frequency similar to the observed frequency. Then, assuming a polar magnetic field strength, $B_{\mathrm{p}}$ of a dipole field, we obtained non-adiabatic eigenfrequency and eigenfunction using the method discussed in [34], in which the expansion was truncated at $J=20$.

We obtain the amplitude modulation and the amplitudes of rotational sidelobes to compare with the observations. A good fit, as presented in Fig. 9, was sought by changing $B_{\mathrm{p}}, \beta$, and $i$ assuming the limb-darkening parameter $\mu=0.6$. Theoretical prediction depends weakly on 

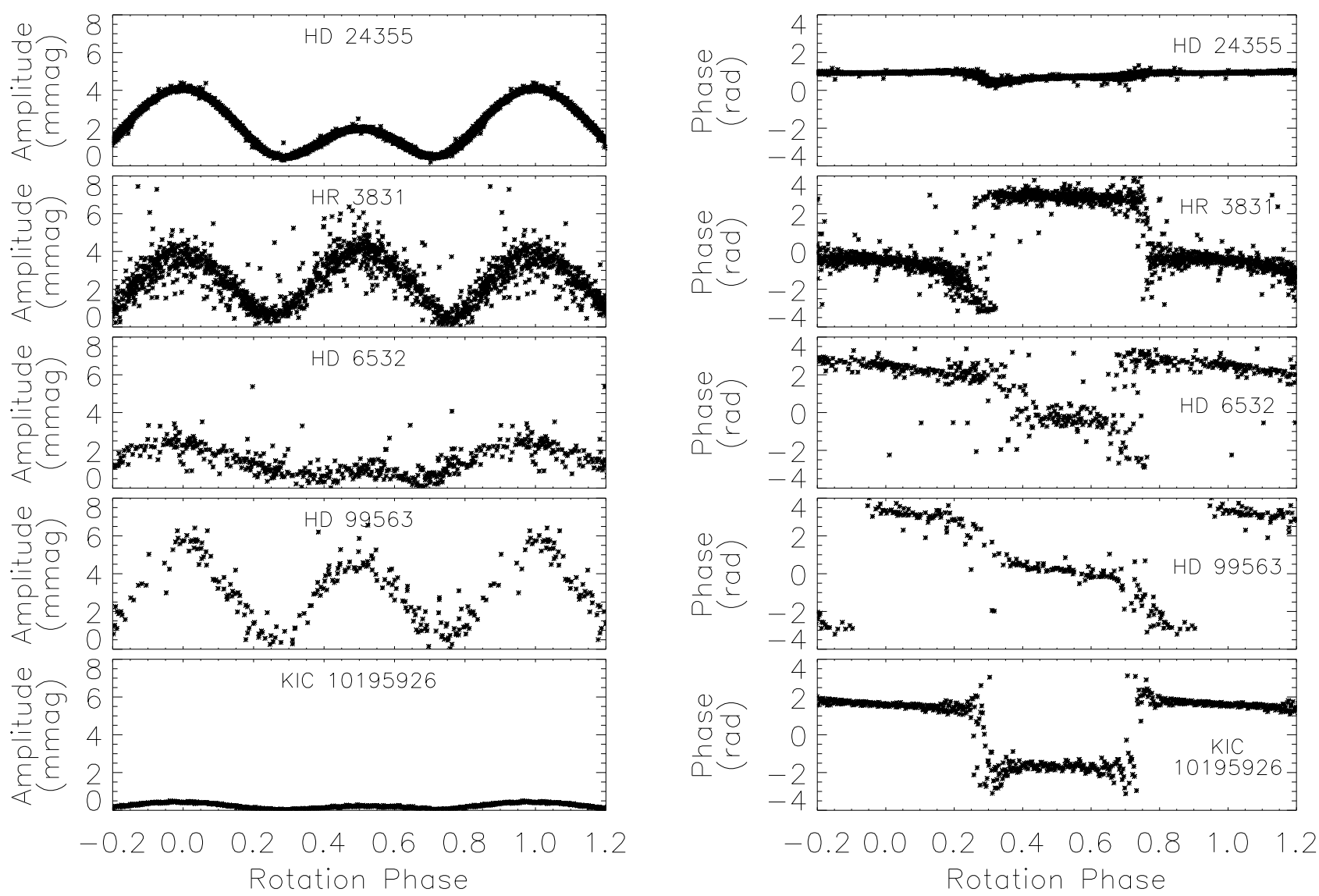

Figure 8: Pulsation amplitude and phase variations as a function of rotation phase for a set of roAp stars which are distorted dipole pulsators. All plots are on the same scale for easy comparison, and have been calculated in the same way. The stars are labelled in the plots. Despite all being distorted dipoles, there is a clear difference between the previously studied stars and HD 24355. Note, the phases are different from those previously published for the comparison stars as a different zero-point was chosen.

the inclination $i$. As our spectroscopic observations allow us to place an upper limit of $i<50^{\circ}$, we present models with $i=45^{\circ}$ as a standard choice, although we can obtain similarly good models in a wide range of $i$.

Although the amplitude modulation of HD 24355 can be reasonably reproduced by models with parameters around the spectroscopically derived values, qualitative properties of the phase modulation systematically change along evolutionary tracks (i.e., $T_{\text {eff }}$ ), as shown in Fig. 10; there is a point where the direction of theoretical phase change switches; the "amplitude" of phase modulation decreases as $T_{\text {eff }}$ decreases. In producing Fig. 10 we ensure that every model in each panel of the figure reproduces well the observed amplitude modulation, as in Fig. 9. We judge that the model in the second panel from the bottom at $\log T_{\text {eff }}=3.910$ best fits with the observed phase modulation among the $2.0 \mathrm{M}_{\odot}$ models. This model has a radius of $2.21 \mathrm{R}_{\odot}$, which gives an upper limit of $i \leq 48^{\circ}$ consistent with $i=45^{\circ}$. We note that a slight asymmetry in observed phase modulation, which cannot be reproduced in our models, is probably caused by a small effect of Coriolis force [11] which we have neglected for simplicity.

Fig. 11 shows the position of HD 24355 in the $\log T_{\text {eff }}-$ $v L / M$ plane together with some of the known roAp stars. In this diagram, the mass dependence of the acoustic cut-off frequency as well as the range of excitation by the $\kappa$-mechanism in the $\mathrm{H}$-ionisation zone [5] are partially compensated for. This figure shows that HD 24355 is a peculiar roAp star whose pulsation is highly supercritical - much more so than for the stars in the well known cooler roAp group consisting of HR 1217, $\alpha$ Cir and $10 \mathrm{Aql}$, etc., all of which are multi-periodic, while HD 24355 is a single-mode pulsator. Another single mode roAp star, HD 42659 [15], could have similar properties to HD 24355, although the position on the diagram depends on the uncertain quantity of $L / M$, and low-amplitude oscillations of HD 42659 are not well studied. The excitation mechanism of such super-critical high-order p-modes is not known.

\section{Conclusions}

We have presented the first Kepler spacecraft observations of the high-amplitude roAp star HD 24355. The K2 data have allowed us to unambiguously determine the rotation period of the star to be $27.9158 \pm 0.0043 \mathrm{~d}$, a parameter which was uncertain when considering the ground-based SuperWASP data alone. Classification dispersion spectra allowed us to classify this star as an $\mathrm{A} 5 \mathrm{Vp} \mathrm{SrEu} \mathrm{star.}$

The high-resolution spectra also allowed us to estimate a mean magnetic field strength of $2.64 \pm 0.49 \mathrm{kG}$; however, 

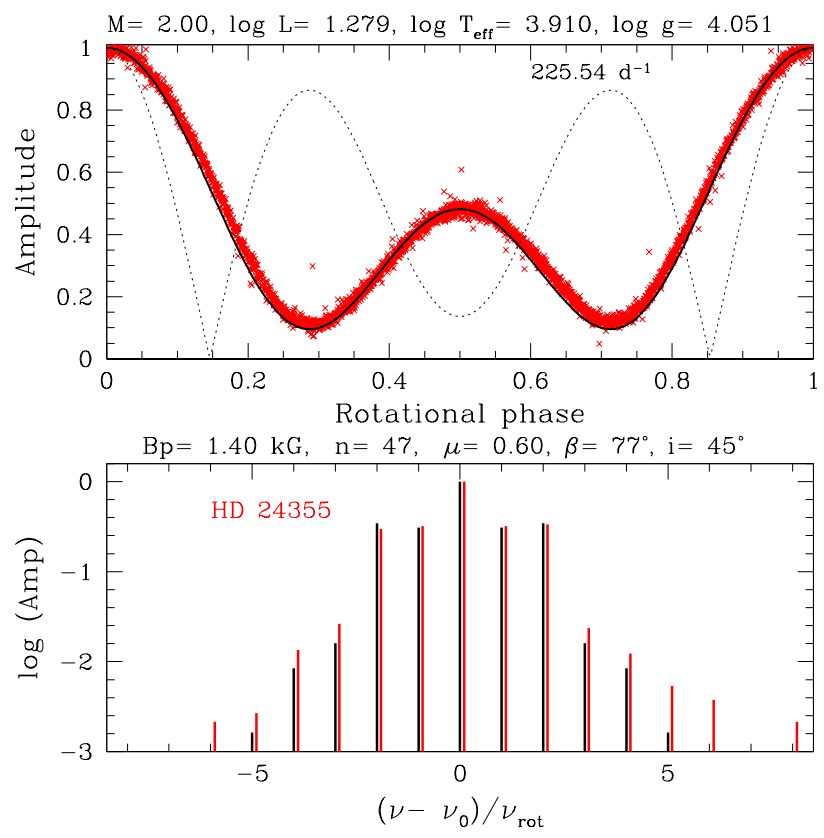

Figure 9: Amplitude modulation (top panel) and corresponding rotational sidelobes (bottom panel) of HD 24355 (red crosses and red bars) are compared with a model (black lines). The dotted line in the top panel shows amplitude modulation expected if the latitudinal distribution of pulsation were proportional to the Legendre function $P_{2}\left(\cos \theta_{B}\right)$. Note that bottom panel plots the logarithmic (base 10) values of sidelobes amplitude normalised by the amplitude of the central frequency.

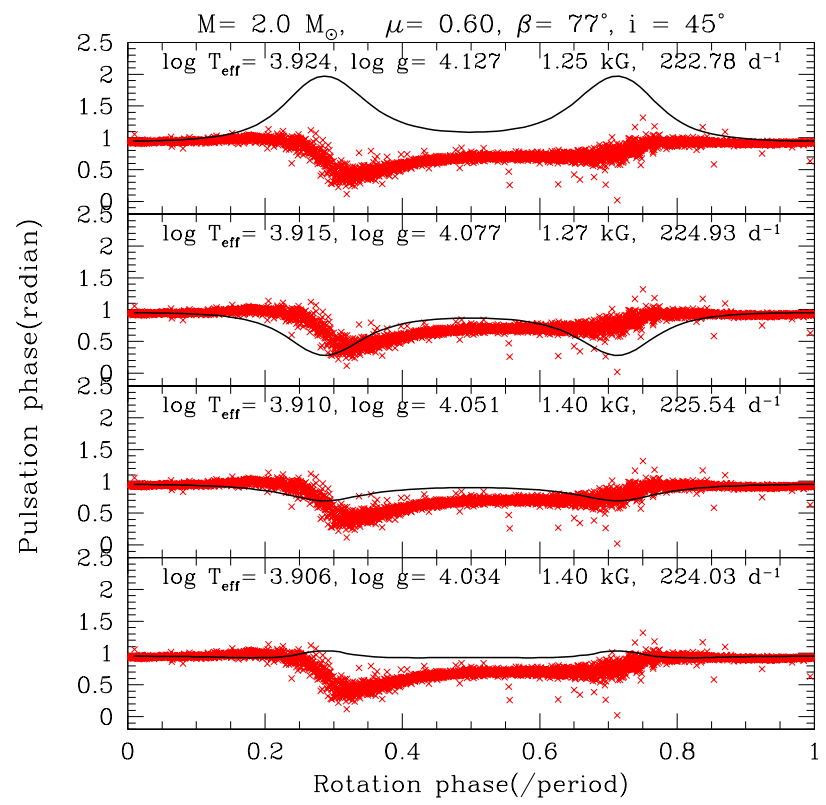

Figure 10: Pulsation phase modulation of HD 24355 (red crosses) with respect to the rotation phase is compared with theoretical ones obtained for some of the evolutionary models of $2 \mathrm{M}_{\odot}$. The effective temperature and surface gravity decrease from top to bottom.

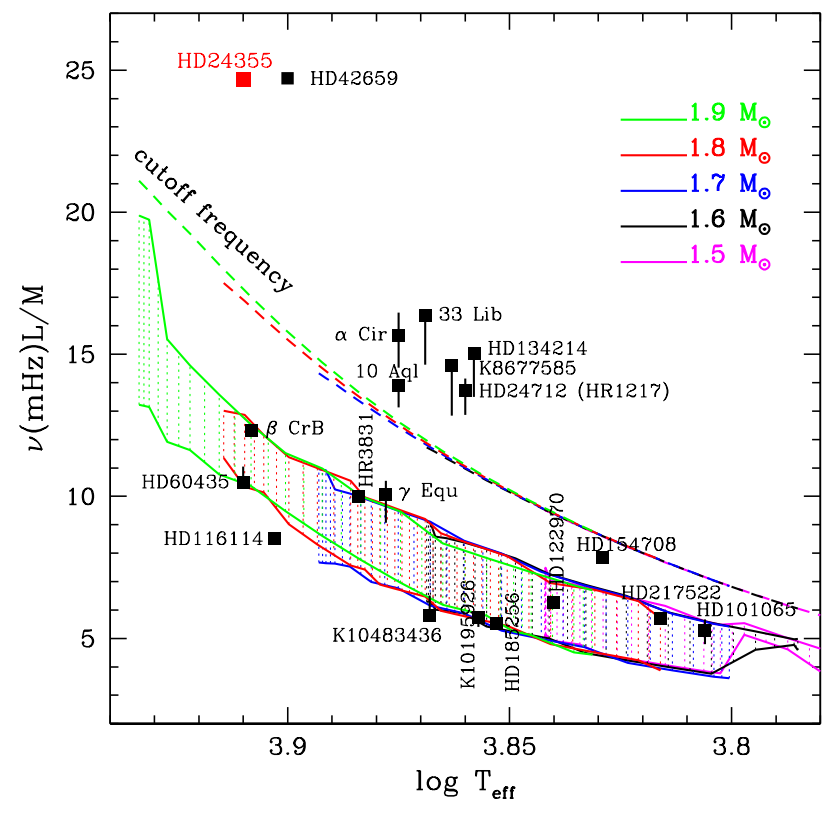

Figure 11: The position of HD 24355 in the $\log T_{\text {eff }}-v L / M$ plane is compared with some of the known roAp stars, in which $L / M$ is in solar units. Main frequencies are shown by squares and frequency ranges by vertical bars. Shaded region indicates where high-order p-modes are excited by the $\kappa$-mechanism in the $\mathrm{H}$ ionisation zone in non-magnetic models. Dashed lines show the acoustic cut-off frequencies. This figure is an extended version of figure 3 in [35].

we take this to be an upper limit on the value due to the lack of Zeeman splitting in the spectra, and the method used to derive that value.

There is a discrepancy between the $T_{\text {eff }}$ of HD 24355 when using different methods to derive the parameter. Values from the literature, SED fitting abundance analysis and line fitting provide a wide range of $T_{\text {eff }}$ values. However, we get the best agreement in results using solely the Balmer lines of both the low-resolution and high-resolution spectra, deriving $8200 \pm 200 \mathrm{~K}$, placing HD 24355 amongst the hotter roAp stars.

Analysis of the pulsation mode as detected in the K2 data have shown the characteristic signatures of a roAp pulsator as predicted by the oblique pulsator model $[3,11,36]$. The pulsational amplitude is modulated with the rotation period of the star. The behaviour of the pulsation phase is not as expected, however. The very small phase change at quadrature is a surprise for a quadrupole pulsator. Examples from the literature (cf. Fig. 8) show a clear $\pi$-rad phase change when a different pulsation pole rotates into view, but this is not the case for HD 24355. Here we see a shift of only 1-rad at most. This small 'blip' in the phase suggests that HD 24355 is pulsating in a very distorted mode, the most extreme case yet observed.

The rotationally split pulsation has provided us with the amplitudes to test the geometry of the star. As such, we modelled the system following the method of [34]. Changing values of the inclination and obliquity angles and the polar magnetic field strength, and searching the parameter space surrounding our observational constraints, we con- 
clude that HD 24355 is a distorted quadrupolar pulsator, with a magnetic field strength of about $1.4 \mathrm{kG}$. The model accurately matches the observed amplitude modulation of the pulsation, and the amplitudes of the rotationally split sidelobes. The pulsational phase variations are a stronger function of the evolution of the star, and as such provide a slightly greater challenge to model. We believe, however, that the model presented is a satisfactory match to the data, given our current observational constraints on the evolutionary stage of the star. We determine that the pulsation seen in HD 24355 is super-critical, making it the most precisely observed super-critical roAp star to date. The driving mechanism for such a pulsation is currently unknown, thus making HD 24355 a highly important target in understanding how some roAp stars can pulsate with frequencies well above the critical cutoff frequency.

\section{References}

[1] D.L. Holdsworth, B. Smalley, M. Gillon, K.I. Clubb, J. Southworth, P.F.L. Maxted, D.R. Anderson, S.C.C. Barros, A.C. Cameron, L. Delrez et al., MNRAS, 439, 2078 (2014)

[2] D.L. Holdsworth, Ph.D. thesis, Keele University (2015)

[3] D.W. Kurtz, MNRAS, 200, 807 (1982)

[4] B. Smalley, E. Niemczura, S.J. Murphy, H. Lehmann, D.W. Kurtz, D.L. Holdsworth, M.S. Cunha, L.A. Balona, M. Briquet, H. Bruntt et al., MNRAS, 452, 3334 (2015)

[5] N.J. Balmforth, M.S. Cunha, N. Dolez, D.O. Gough, S. Vauclair, MNRAS, 323, 362 (2001)

[6] M.S. Cunha, D. Alentiev, I.M. Brandão, K. Perraut, MNRAS, 436, 1639 (2013)

[7] H. Shibahashi, H. Saio, PASJ, 37, 245 (1985)

[8] H. Shibahashi, H. Saio, PASJ, 37, 601 (1985)

[9] H. Shibahashi, M. Takata, PASJ, 45, 617 (1993)

[10] M. Takata, H. Shibahashi, PASJ, 47, 219 (1995)

[11] L. Bigot, D.W. Kurtz, A\&A, 536, A73 (2011)

[12] H.W. Babcock, ApJ, 132, 521 (1960)

[13] T. Lüftinger, O. Kochukhov, T. Ryabchikova, N. Piskunov, W.W. Weiss, I. Ilyin, A\&A, 509, A71 (2010)

[14] P. Martinez, D.W. Kurtz, G.M. Kauffmann, MNRAS, 250, 666 (1991)

[15] P. Martinez, D.W. Kurtz, MNRAS, 271, 118 (1994)

[16] I.S. Savanov, V.P. Malanushenko, T.A. Ryabchikova, Astronomy Letters 25, 802 (1999)

[17] O. Kochukhov, T. Ryabchikova, A\&A, 374, 615 (2001)

[18] A.P. Hatzes, D.E. Mkrtichian, MNRAS, 351, 663 (2004)
[19] D.E. Mkrtichian, A.P. Hatzes, H. Saio, R.R. Shobbrook, A\&A, 490, 1109 (2008)

[20] V.G. Elkin, D.W. Kurtz, G. Mathys, MNRAS, 415, 2233 (2011)

[21] O. Kochukhov, D. Alentiev, T. Ryabchikova, S. Boyko, M. Cunha, V. Tsymbal, W. Weiss, MNRAS, 431, 2808 (2013)

[22] D.L. Pollacco, I. Skillen, A. Collier Cameron, D.J. Christian, C. Hellier, J. Irwin, T.A. Lister, R.A. Street, R.G. West, D.R. Anderson et al., PASP, 118, 1407 (2006)

[23] L.A. Balona, M.S. Cunha, D.W. Kurtz, I.M. Brandão, M. Gruberbauer, H. Saio, R. Östensen, V.G. Elkin, W.J. Borucki, J. Christensen-Dalsgaard et al., MNRAS, 410, 517 (2011)

[24] L.A. Balona, M.S. Cunha, M. Gruberbauer, D.W. Kurtz, H. Saio, T.R. White, J. ChristensenDalsgaard, H. Kjeldsen, J.L. Christiansen, J.R. Hall et al., MNRAS, 413, 2651 (2011)

[25] D.W. Kurtz, M.S. Cunha, H. Saio, L. Bigot, L.A. Balona, V.G. Elkin, H. Shibahashi, I.M. Brandão, K. Uytterhoeven, S. Frandsen et al., MNRAS, 414, 2550 (2011)

[26] D.L. Holdsworth, B. Smalley, D.W. Kurtz, J. Southworth, M.S. Cunha, K.I. Clubb, MNRAS, 443, 2049 (2014)

[27] X.Q. Cui, Y.H. Zhao, Y.Q. Chu, G.P. Li, Q. Li, L.P. Zhang, H.J. Su, Z.Q. Yao, Y.N. Wang, X.Z. Xing et al., Research in Astronomy and Astrophysics 12, 1197 (2012)

[28] G. Zhao, Y.H. Zhao, Y.Q. Chu, Y.P. Jing, L.C. Deng, Research in Astronomy and Astrophysics 12, 723 (2012)

[29] A.L. Luo, Y.H. Zhao, G. Zhao, L.C. Deng, X.W. Liu, Y.P. Jing, G. Wang, H.T. Zhang, J.R. Shi, X.Q. Cui et al., Research in Astronomy and Astrophysics 15, 1095 (2015)

[30] R.O. Gray, C. Corbally, J., Stellar Spectral Classification (2009)

[31] D.L. Holdsworth, D.W. Kurtz, B. Smalley, H. Saio, G. Handler, S.J. Murphy, H. Lehmann, MNRAS, 462, 876 (2016)

[32] M. Still, T. Barclay, PyKE: Reduction and analysis of Kepler Simple Aperture Photometry data, Astrophysics Source Code Library (2012)

[33] D.W. Kurtz, H. Shibahashi, P.R. Goode, MNRAS, 247, 558 (1990)

[34] H. Saio, MNRAS, 360, 1022 (2005)

[35] H. Saio, Pulsation of magnetic stars, in Precision Asteroseismology, edited by J.A. Guzik, W.J. Chaplin, G. Handler, A. Pigulski (2014), Vol. 301 of IAU Symposium, pp. 197-204

[36] L. Bigot, W.A. Dziembowski, A\&A, 391, 235 (2002) 